\title{
Metastable bound state of two likely charged classic Coulomb particles
}

\author{
Alexander E. Dubinov, Ilya N. Kitayev \\ Sarov Institute of Physics and Technology (SarFTI), National Research Nuclear University "MEPhI," Sarov, Nizhni Novgorod region, \\ 607188, Russia
}

\section{Email address:}

dubinov-ae@yandex.ru (A. E. Dubinov),rmtmkitaeva@yandex.ru (I. N. Kitayev)

\section{To cite this article:}

Alexander E. Dubinov, Ilya N. Kitayev. Metastable Bound State of Likely Charged Classic Coulomb Particles. American Journal of Modern Physics. Vol. 2, No. 2, 2013, pp. 53-57. doi: 10.11648/j.ajmp.20130202.13

\begin{abstract}
When the classical charged particle is scattered on the likely charged target particle, executing the rapid finite motion, a metastable bound state of the incident particle and the target particle may occur. This type of behavior differs dramatically from the Rutherford scattering.
\end{abstract}

Keywords: Charged particle; Finite motion; Metastable bound state; Scattering

\section{Introduction}

According to classical physics, the likely charged particles are always repelled from each others and can not form a bound state. This was a statement, which no one could ever prejudice before. However, in the course of time it was found out, that certain physical states exist, at which two likely charged particles are effectively attracted, and as a result, a stable bound state or a pair is formed.

Cooper pairing of degenerated electrons, occurring in the crystal lattice thanks to polarizing electron-phonon interaction [1] is the first example of the above said attraction. It is worth reminding that the phenomenon of Cooper pairing is fundamental for understanding of super-conductivity of metals.

Let us recall several recent examples of similar coupling mechanisms. In [2], for instance, interaction of two electrons in low-temperature plasma via virtual acoustic waves is considered. It is shown, that under certain conditions electrons can be attracted to each other. This type of attraction, as is assumed in [2], allows to explain stability and big lifetime of compact plasmoids such as ball lightning. As is shown in [3], when plasma, featuring quantum-degenerated electrons is calculated, effective attraction of likely charged ions at certain distances occur, if electron-correlation potential is accounted for, which, in its turn, may result in phase transitions occurring in quantum plasma. Examples [1-3] may be referred to the type of bounding, which becomes possible due to collective interaction with background medium.
However, alternative types of bounding and attraction of likely charged particles may be exemplified here. In papers [4-6], for instance, electrostatic interaction of two closely located charged metal balls of different diameter is considered. The idea of attraction of these balls is based on inhomogeneous distribution of charges along the surface of the balls: as the charges are repelled from each other, they relocate to the remote sections of the ball surface, leaving non-compensated charged of the opposite sign at the proximal sections. As a result, two sections with different signs of charge are formed on the surface of both balls, due to which attraction is ensured and formation of electrostatically bound state of the balls is stipulated for.

The present paper offers a new principle of creation of the bound state for the likely charged particles, based on the dynamic capture, happening when one particle is scattered on the other particle.

\section{Calculation of A Metastable Bound State}

It is known, that the process of scattering of the incident particle on the other particle, which initially plays the role of immobile target, obeys the Rutherford law [7]. However, if the target particle, for any reason, executes the rapid finite motion: oscillates, rotates or moves along some other closed trajectory, than the Rutherford scattering law is not satisfied and in certain cases a long-living bound state between the incident particle and the target particle may be formed. Presented below are some examples of this state- 
ment.

Let us consider the simple case of scattering of the incident particle on the target particle in a plane setup. We will assume that the target particle executes some specified finite motion according to the following law:

$$
\left\{\begin{array}{l}
\bar{x}=x_{0} \cos \left(\omega_{1} t+\varphi_{1}\right) ; \\
\bar{y}=y_{0} \sin \left(\omega_{2} t+\varphi_{2}\right) .
\end{array}\right.
$$

Then, in general case, its trajectory will be elliptic. Having assumed that the incident particle and the target particle interact according to the Coulomb force, one may match the parameters of interaction in such a way, that the incident particle will stay inside the above ellipse arbitrarily long.

Figure 1 illustrates the trajectory of the incident particle, locked inside the elliptic trajectory of the target particle, calculated as the solution of the classic motion equation and written in the coordinate form as the system of normalized differential equations:

$$
\left\{\begin{array}{l}
\frac{d^{2} x}{d t^{2}}=\frac{x-\bar{x}}{\left[(x-\bar{x})^{2}+(y-\bar{y})^{2}\right]^{3 / 2}} \\
\frac{d^{2} y}{d t^{2}}=\frac{y-\bar{y}}{\left[(x-\bar{x})^{2}+(y-\bar{y})^{2}\right]^{3 / 2}} .
\end{array}\right.
$$

Figure 1 testified to the fact, that the target particle forms the effective dynamic potential well, the contour of which is provided by its elliptic trajectory. Parameters of the corresponding calculation are as follows: $x_{0}=y_{0}=1 ; \omega_{1,2}=10 \pi$; $\varphi_{1}=5 \pi / 4 ; \varphi_{2}=-3 \pi / 2$, initial velocity of the incident particle has components

$$
\left\{\begin{array}{l}
v_{x}(0)=2.21196099999 ; \\
v_{y}(0)=0,
\end{array}\right.
$$

and its initial disposition

$$
\left\{\begin{array}{l}
x(0)=-9.9 ; \\
y(0)=0.108000009 .
\end{array} .\right.
$$

Figure 2 shows the time dependence of the distance $r(t)$ between the instantaneous position of the incident locked particle and the origin of coordinates, demonstrating that the process of containment may last long enough, so that one might conclude that metastable bound state of the two likely charged classic particles have formed. The incident particle is locked and retained as follows: as soon as the incident particle approximates the ellipse, the rapidly rotating target particle finds itself there and pushes the incident particle into the ellipse. This mechanism is similar to one-dimensional locking of the charged particle by an oscillating potential barrier, which is described in $[8,9]$.

Such solutions may be observed at the same elliptic trajectory pf the target particle, but at other initial conditions. For example, metastable bound state at

$$
\left\{\begin{array}{l}
v_{x}(0)=2.2099949996 \quad 93 ; \\
v_{y}(0)=0,
\end{array}\right.
$$

and

$$
\left\{\begin{array}{l}
x(0)=-9.9990098518 \quad 9 \\
y(0)=0.0987000038 \quad 001 .
\end{array}\right.
$$

it is presented in Figure 3 and 4.

As the calculations show, the above capture of the incident particle into the bound state may repeat in the other finite trajectories of the target particle, for instance, the Lissajous curves. Results of the calculations with the Lissajous curves are shown in Figure 5-7: for $x_{0}=y_{0}=1$; $\omega_{1}=16 \pi ; \omega_{2}=24 \pi ; \varphi_{1}=5 \pi / 4 ; \varphi_{2}=-3 \pi / 2$ at initial conditions

$$
\left\{\begin{array}{l}
v_{x}(0)=2.2209994999693 \\
v_{y}(0)=0,
\end{array}\right.
$$

and

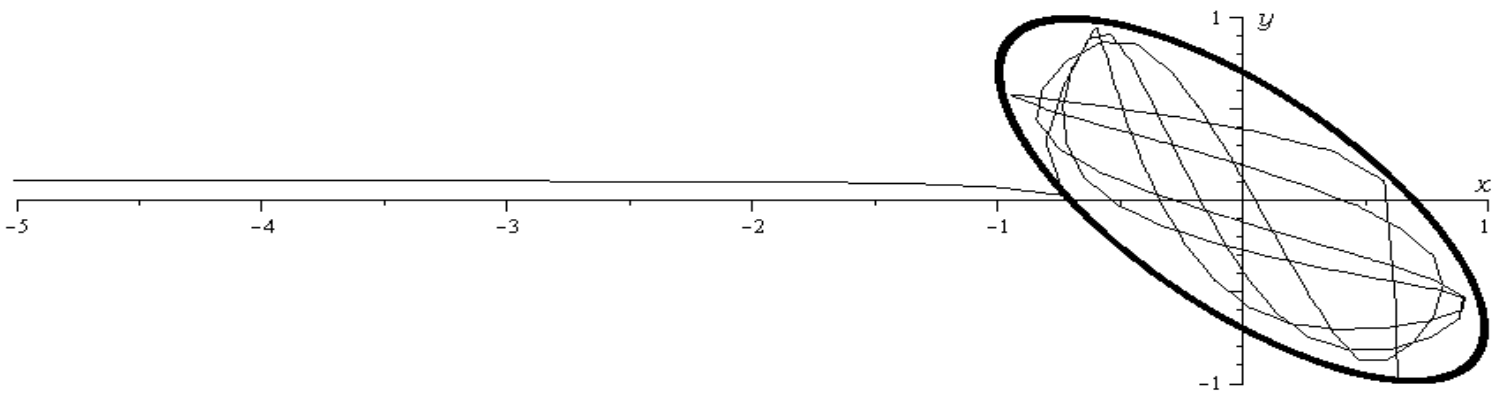

Figure 1. Trajectories of the particles: thick line for the target particle; thin line for the incident particle at initial conditions (3) and (4). 


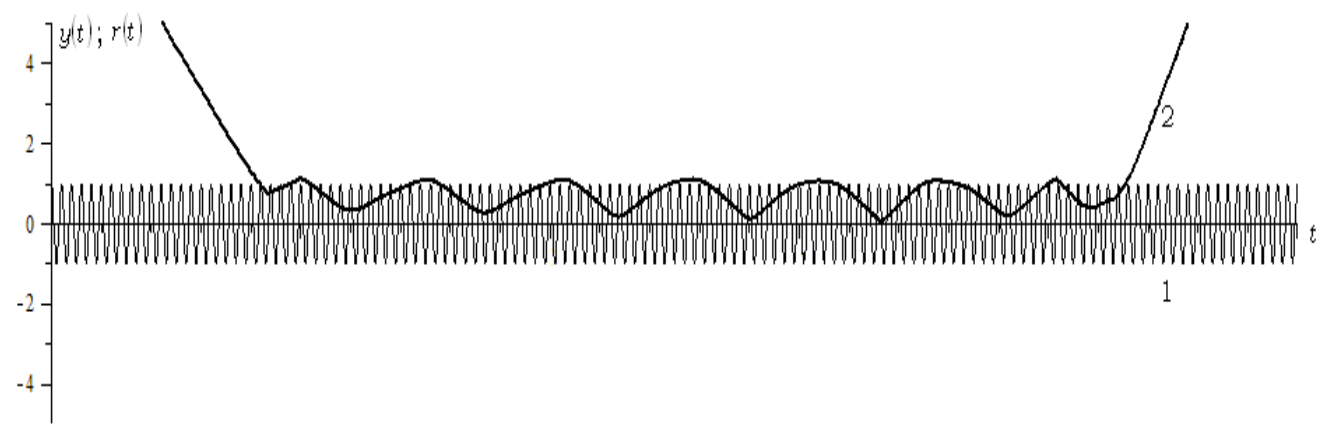

Figure 2. The time dependences of transverse coordinate for the target particle (1) and of distance between position of the incident locked particle and the origin of coordinates at initial conditions (3) and (4).

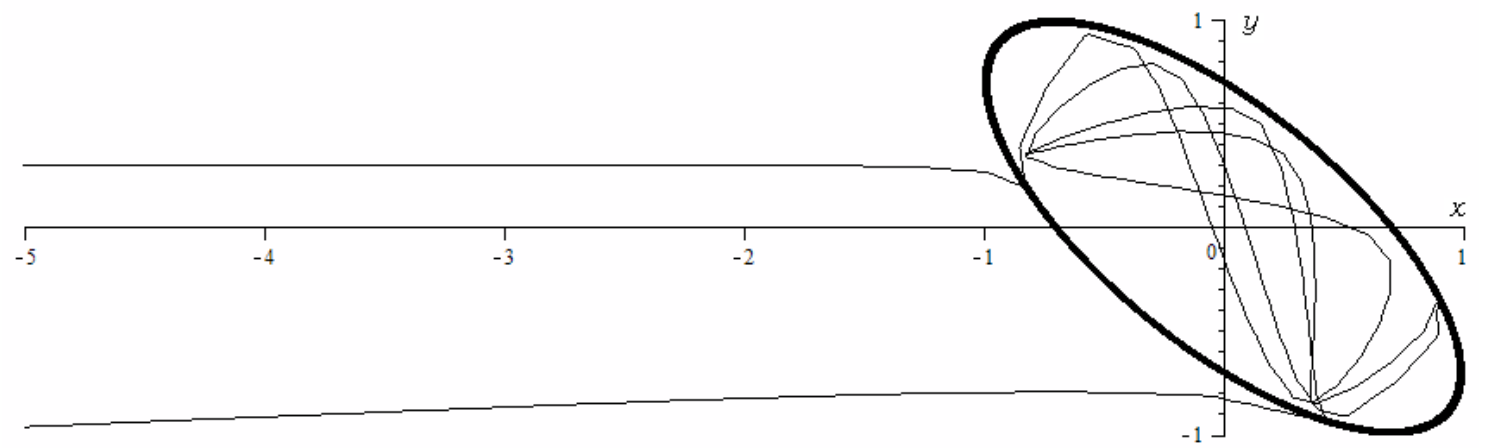

Figure 3. Trajectories of the particles: thick line for the target particle; thin line for the incident particle at initial conditions (5) and (6).

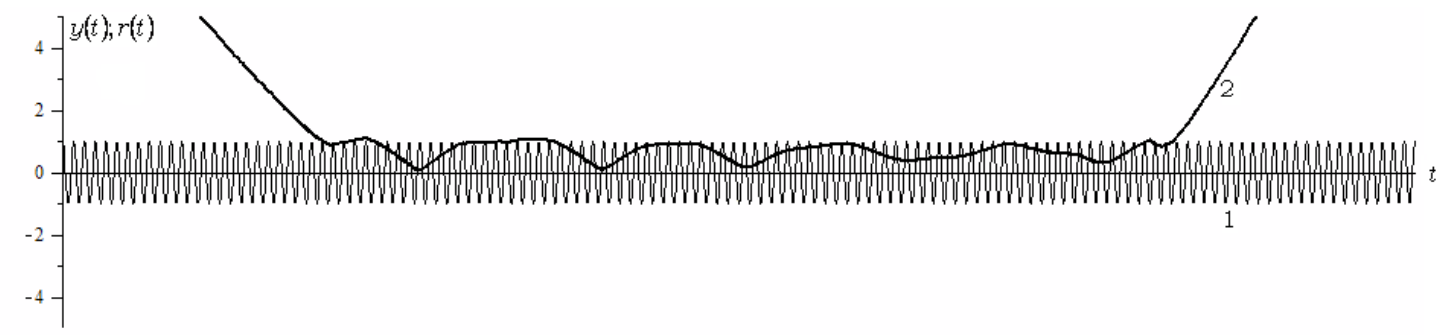

Figure 4. The time dependences of transverse coordinate for the target particle (1) and of distance between position of the incident locked particle and the origin of coordinates at initial conditions (5) and (6).

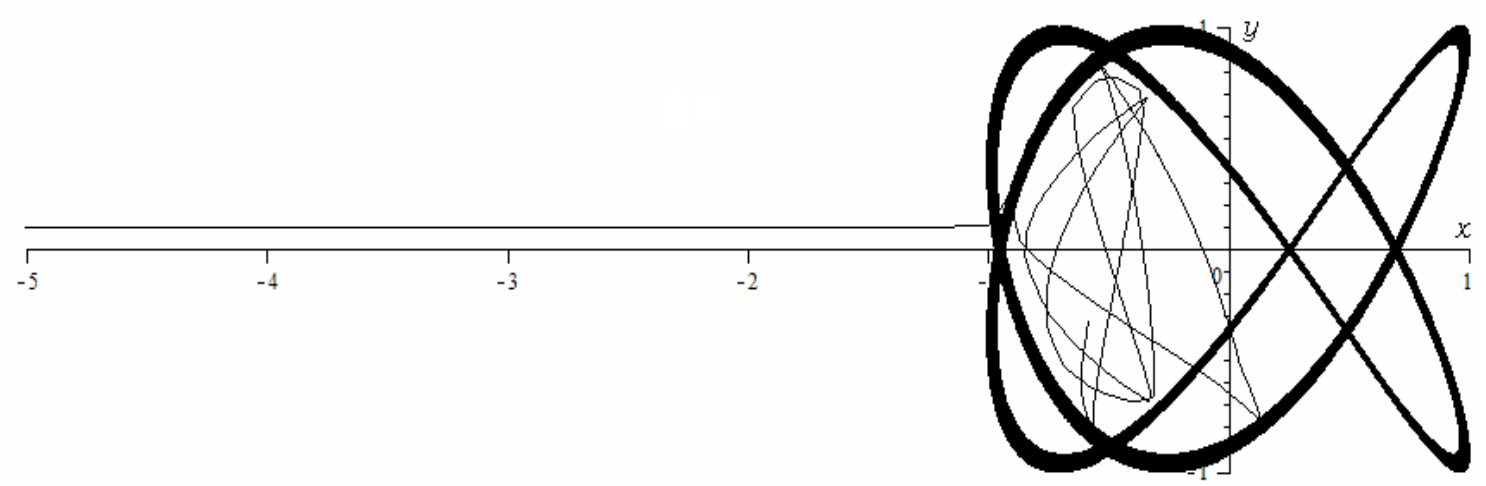

Figure 5. Trajectories of the particles: thick line for the target particle; thin line for the incident particle at initial conditions (7) and (8). 


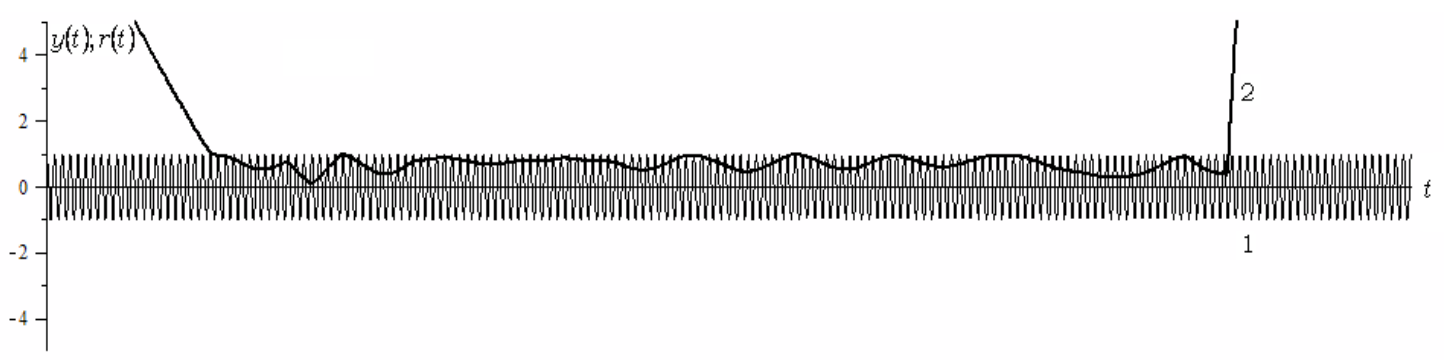

Figure 6. The time dependences of transverse coordinate for the target particle (1) and of distance between position of the incident locked particle and the origin of coordinates at initial conditions (7) and (8).

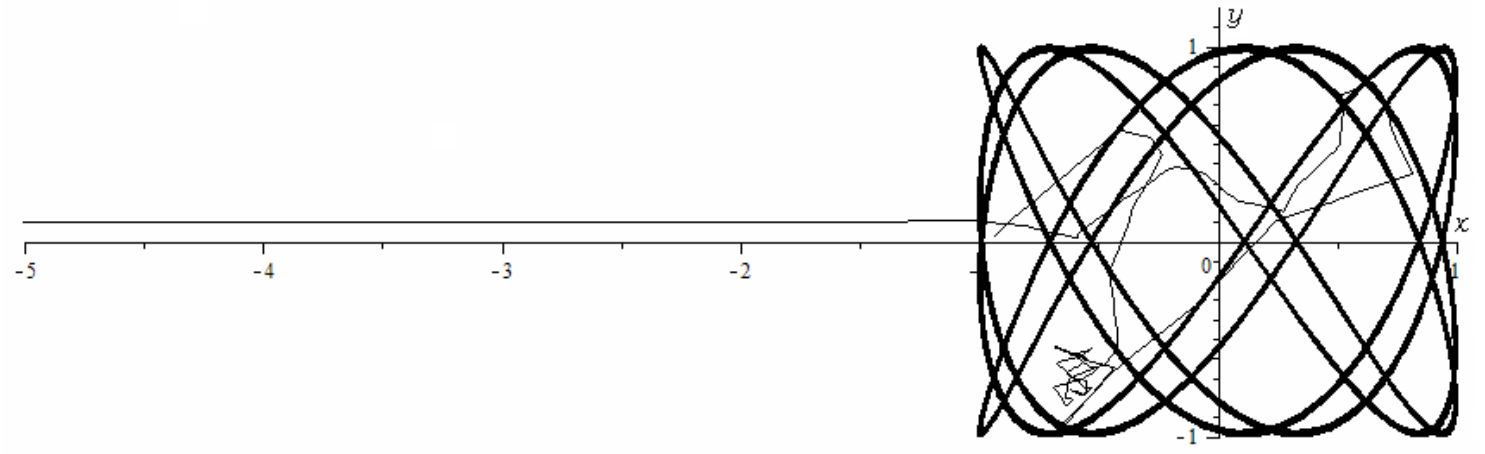

Figure 7. Trajectories of the particles: thick line for the target particle; thin line for the incident particle at initial conditions (9) and (10) .

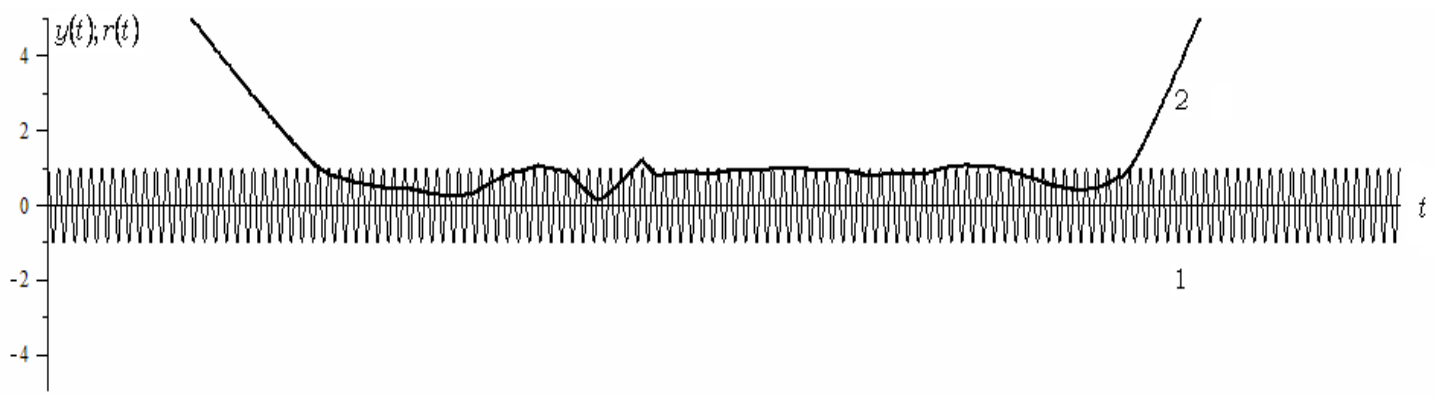

Figure 8. The time dependences of transverse coordinate for the target particle (1) and of distance between position of the incident locked particle and the origin of coordinates at initial conditions (9) and (10).

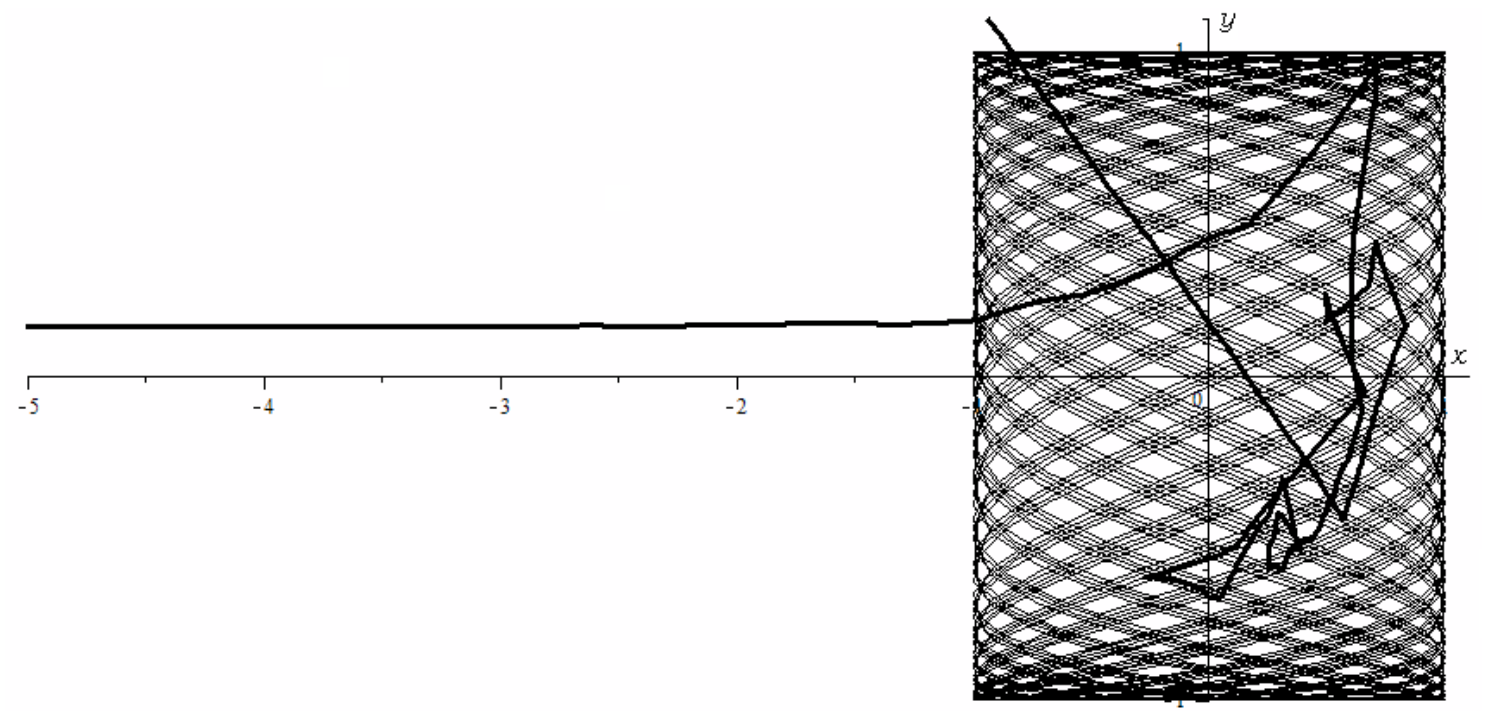

Figure 9. Trajectories of the particles: thick line for the target particle; thin line for the incident particle at initial conditions (11) and (12). 


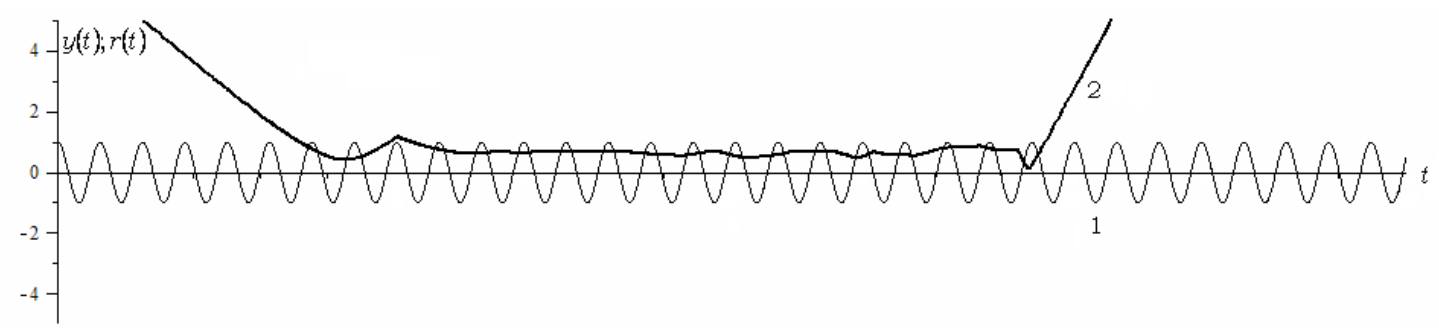

Figure 10. The time dependences of transverse coordinate for the target particle (1) and of distance between position of the incident locked particle and the origin of coordinates at initial conditions (11) and (12).

$$
\left\{\begin{array}{l}
x(0)=-9.9990098 ; \\
y(0)=0.2990000909 \quad 9,
\end{array}\right.
$$

and for other Lissajous curves for $x_{0}=y_{0}=1 ; \omega_{1}=8 \pi$; $\omega_{2}=14 \pi ; \varphi_{1}=5 \pi / 4 ; \varphi_{2}=-3 \pi / 2$ at

$$
\left\{\begin{array}{l}
v_{x}(0)=1.9012101249 \quad 154 ; \\
v_{y}(0)=0,
\end{array}\right.
$$

and

$$
\left\{\begin{array}{l}
x(0)=-9.9999002500 \quad 07 ; \\
y(0)=0.0987020300 \quad 69999
\end{array} .\right.
$$

We have considered still only closed finite trajectories of the target particle. Then one example of open finite trajectory is shown in the Figures 9 and 10. The figures are drawn for next parameters: $x_{0}=y_{0}=1 ; \omega_{1}=10 \pi ; \omega_{2}=10$; $\varphi_{1}=5 \pi / 4 ; \varphi_{2}=-3 \pi / 2$ at

$$
\left\{\begin{array}{l}
\mathrm{v}_{\mathrm{x}}(0)=1.8999196346002 ; \\
\mathrm{v}_{\mathrm{y}}(0)=0,
\end{array}\right.
$$

and

$$
\left\{\begin{array}{l}
x(0)=-7.400010949818 \\
y(0)=0.150000943000206 .
\end{array}\right.
$$

\section{Conclusions}

Thus, a possibility of formation of the bound state of the two likely charged classic particles is demonstrated and proved via calculations in the present paper for different finite trajectories of the target particle.

\section{Acknowledgements}

The activities were executed under the auspices of Russian-Ukrainian Grant RFBR \#12-02-90433-Ukr_a.

\section{References}

[1] Cooper L. N., Bound electron pairs in a degenerate Fermi gas. Phys. Rev., 104(4), pp. 1189-1190, 1956.

[2] Dvornikov M., Effective attraction between oscillating electrons in a plasmoid via acoustic wave exchange. Proc. Royal Soc. A, 468(2138), pp. 415-428, 2012.

[3] Shukla P. K., and Eliasson B., Novel attractive force between ions in quantum plasmas. Phys. Rev. Lett., 108(16), pp. $165007-1-5,2012$.

[4] Lekner J., Analytical expression for the electric field enhancement between two closely-spaced conducting spheres. J. Electrostatics, 68(4), pp. 299-304, 2010.

[5] Lekner J., Electrostatics of two charged conducting spheres. Proc. Royal Soc. A, 468(2145), pp. 2829-2848, 2012.

[6] Lekner J., Electrostatic force between two conducting spheres at constant potential difference. J. Appl. Phys., 111(7), pp. 076102-1-2, 2012.

[7] Landau L. D., Lifshitz E. M., Mechanics, 3rd ed. Butterworth-Heinemann, Oxford, 1976.

[8] Dubinov A. E., Saikov S. K., and Selemir V. D., On dynamics of metastable electrons in a virtual cathode. Radiophysics and Quantum Electronics, 45(4), pp. 318-321, 2002

[9] Dubinov A. E., Saikov S. K., Metastable balancing oscillators. Plasma. Phys. Rep., 28(5), pp. 398-402, 2002. 